\title{
Review of: "RUNX1 and REXO2 are associated with the heterogeneity and prognosis of IDH wild type lower grade glioma"
}

Weiguo Zhang

Potential competing interests: The author(s) declared that no potential competing interests exist.

The combination of sequencing technology and clinical practice has established a series of molecular markers related to the tumorigenesis, progression, treatment and prognosis of glioma, which leads to improvements in the lives of patients with glioma. In this study, the authors enriched the molecular characteristics of glioma and provided novel prognostic makers to predict the clinical outcomes of IDH wild type LGG by analyzing the transcriptional data of 131 cases of LGG. They found that RUNX1 and its target gene REXO2 were associated with the poor prognosis and immune alterations in IDH wild type LGG, which has significant clinical value. However, as stated by the authors, the expression and prognosis of RUNX1 and REXO2 in large cohorts of IDH wild type LGG should be studied. 